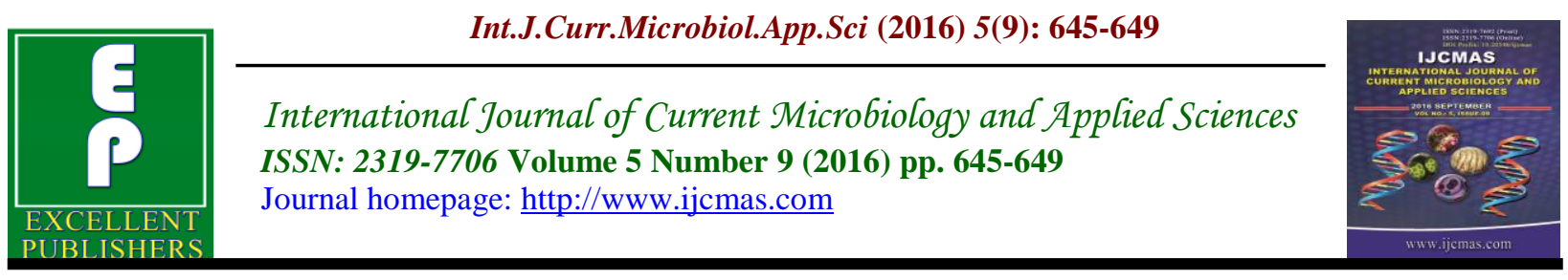

Original Research Article

http://dx.doi.org/10.20546/ijcmas.2016.509.074

\title{
Bacteriological Study of Central Line Associated Blood Stream Infection at a Tertiary Care Hospital
}

\author{
G. Naveen*, G. Latha and C. Nagraj \\ Department of Microbiology, PES institute of Medical Sciences and Research, \\ Kuppam - 517425, Chittoor, Andhra Pradesh, India \\ *Corresponding author
}

Keywords

Central line associated blood stream infection, ICU, antimicrobial sensitivity testing.

\section{Article Info}

Accepted:

25 August 2016

Available Online:

10 September 2016

\section{A B S T R A C T}

Central venous catheter insertion is very common in ICU settings and is one of the serious and important causes for health care associated infections. We conducted this study to find out the bacteriological profile and antimicrobial susceptibility pattern of the isolates in central line associated blood stream infections in the ICU practice over a duration of 2 years. Patients admitted in ICU settings requiring central venous catheterization and presenting with signs of septicemia were investigated for catheter associated blood stream infections. The peripheral blood and the tip of central venous catheter were obtained simultaneously and processed in the microbiology lab. The isolated organisms were subjected for antimicrobial susceptibility testing. There were 11 episodes of central line associated blood stream infections of which $3(27.27 \%)$ were due to Pseudomonas aeruginosa, 3 (27.27\%) due to Acinetobacter species, 2 (18.18\%) due to Staphylococcus aureus, $2(18.18 \%)$ due to Klebsiella species and 1 (9\%) due to Staphylococcus epidermidis. Central line associated blood stream infection rate of 22.6/1000 catheter days was observed in this study. Regular surveillance of catheter associated blood stream infection is required for better patient outcome as central venous catheter are increasingly being used at ICU.

\section{Introduction}

Central venous catheter insertion is one of the key factors for modern medical practice, however insertion process are significantly associated with iatrogenic diseases, especially bacteremia (Mermel et al., 2000). Catheter associated blood stream infection is defined as the presence of bacteremia arising from an intravenous catheter (Brun-Buisson et al., 1987). Gram negative bacilli are been on a rise as a causative agent of catheter related infection in ICU patient. The preventive measures like using local antibiotics, antimicrobial impregnated catheters and maximal sterile barrier precautions following have shown to reduce the rate of catheter associated blood stream infection (Karpel et al., 2006). In this study, we planned to find the incidence, bacteriological causes, and the antimicrobial sensitivity pattern of the isolates from central line associated blood stream infections. 


\section{Materials and Methods}

The present study was conducted in a tertiary care hospital, PESIMSR, Kuppam over duration of 2 years. This study included patients admitted in adult ICU requiring central venous catheterization and having signs and symptoms of septicemia 48 hours after insertion of central venous catheter.

Brief epidemiological data and personal data were collected from the patients or patient's attenders and the clinical data was taken from concern medical staff on a predesigned form, containing important data as well as laboratory investigation reports. A total of 59 central line tips were collected. From these catheters the distal $5 \mathrm{~cm}$ was cut with sterile blade and transported in a sterile container. Peripheral blood was collected with proper aseptic precautions and sent along with the collected catheter. Catheter tip was processed and reported according to determined protocol (Brun-Buisson C et al., 1987). From the inoculated blood culture bottles few drops of media were taken after gentle agitation and subcultured on blood agar and Macconkey agar followed by incubating overnight at $37^{\circ} \mathrm{C}$. Next morning, the culture plates were examined and colony morphology was noted. Blood culture was reported negative after 7 days of incubation. Culture isolates were identified as per standard microbiological methods followed in the laboratory and antimicrobial susceptibility testing was done by KirbyBaur disc diffusion technique according to CLSI guidelines (CLSI, 2003, CLSI, 2008). For reporting of the laboratory results, the following definitions were followed:

\section{Catheter associated blood stream infection}

A positive blood culture with the same organism isolated from central venous catheter tip culture was considered to indicate catheter associated blood stream infection.

\section{Blood stream infection}

A positive blood culture with a negative central venous catheter tip culture or a different organism isolated from central venous catheter tip was assumed to be a blood stream infection (CDC/NHSN, 2013).

The rate of catheter associated blood stream infection was expressed in number of central venous catheter days and was calculated by the following formula:

Central line associated blood stream infection rate per 1000 catheter days

$=\underline{\text { No. Of central line associated blood stream infection cases }} \times 1000$.

No. Central line catheter days

\section{Results and Discussion}

In the present study, the duration of catheter insertion range from 4 to 48 days with a mean of 7.6 days and a total of 487 days. Out of 59 patients, we found catheter associated blood stream infection rate of $22.6 / 1000$ catheter days and $18.6 \%$ as the incidence of central line associated blood stream infection. We found increase in central line catheter colonization rate with increased duration of catheterization. We found 14 blood cultures positive out of 59 samples among which 4 were blood stream infections. We found all central line associated blood stream infection (CLBSI) were associated with central venous catheter colonization. In the present study out of 11 
CLBSI causing organisms $3(27.7 \%)$ were due to Pseudomonas aeruginosa, 3 (27.7\%) due to Acinetobacter species, 2 (18.18\%) due to Staphylococcus aureus, 2 (18.18\%) due to Klebsiella species, and 1 (9\%) due to Staphylococcus epidermidis. In the present study, we found gram negative bacilli to be the more common causative agents compare to gram positive cocci.

Central line associated blood stream infection may result from healthcare interventions and constitutes an important cause of morbidity and mortality among patients in critical care settings. Various factors may contribute for the role of bacterial colonization in the catheter such as poor personal hygiene, moisture around the exit site, occlusive dressings, Staphylococcus aureus, nasal colonization and poor hand hygiene. The incidence rate of central line catheter colonization of various studies ranges from $31.58 \%$ to $76 \%$ (Meadows et al., 2009; Tullu et al., 1998; Karpel et al., 2006). In all other studies, gram positive cocci were the predominant colonizers of central venous catheter, but in our study, we found gram negative bacilli like Pseudomonas aeruginosa, Acinetobacter species, Klebsiella species to be the predominant ones compare to gram positive cocci like Staphylococcus species. Organisms like Pseudomonas aeruginosa and Acinetobacter species which are saprophytes favouring moist environment could be the causative agents when there is moisture around the exit sites.

The incidence of central associated blood stream infection was $18.6 \%$. This is higher than that of National Nosocomial Infections Surveillance (NNIS, 2004) System Report (1.8 to 5.2 per 1,000 catheter days). Our study matched the infection rate incidence of Yilmaz et al., (Yilmaz et al., 2007) and Almuneef et al., (Almuneef et al., 2006). A study by Yilmaz et al., (Yilmaz et al., 2007) showed that in their study period, 111 intravascular catheters through which parenteral nutrition was administered and monitored for a total of 1646 catheter-days showed CLBSI rate of 18.8 per 1,000 catheter-days. Similar findings were seen by Almuneef et al., (Almuneef et al., 2006) who found infection rate of 20 per 1000 catheter days in his study. The duration of catheterization is a significant factor that determined the development of catheter related infections (Richet et al., 1990; Heard et al., 1998; Moro et al., 1994; Gil et al., 1989). Although previous studies have confirmed that central venous catheterization longer than 5 days was associated with a higher risk of catheter related infection, the mean duration of catheterization in our study of 7.6 and no attempts were made to replace catheter as per the CDC guidelines of 1996 and 2002 recommendations. This could probably be the cause for slightly higher infection rates in our study. The other reason for slightly elevated rates of infections could be due to not strictly adhering to preventive bundles due to various reasons.

In conclusion, central line associated blood stream infection remains an important complication of central venous access in ICU's. The duration of catheterization and colonization has an important role in the development of central line associated blood stream infection along with various other causes which may lead to septicemia and multiorgan failure. Central line associated blood stream infection should be suspected in all the catheterized patients having signs and symptoms of septicemia. Local antibiotics strict preventive bundles and catheter lock solutions should be used as preventive measure while inserting the central line catheter. Long duration of catheterization increases the morbidity and 
mortality, so regular monitoring of catheterized patients should be done for both insertion site and general condition. Culture and sensitivity pattern can be a very good guide to treat specific organism as most isolates are resistant to commonly used antibiotics. This may also be accompanied by removal of the catheter to reduce the mortality and morbidity from central line associated blood stream infection, thus producing better patient care and outcome.

\section{Acknowledgement}

We are thankful to the whole infection control team, intensivist and quality team (QMS) of PESIMSR, Kuppam in bringing out this study.

\section{References}

Almuneef, M.A., Memish, Z.A., Balkhy, H.H., Hijazi, O., Cunningham, G., Francis, C. 2006. Rate, risk factors and outcomes of catheter-related bloodstream infection in a paediatric intensive care unit in Saudi Arabia. $J$. Hosp. Infect., 62: 207-213.

Brun-Buisson, C., Abrouk, F., Legrand, P., Huet, Y., Larabi, S., Rapin, M. 1987. Diagnosis of central venous catheterrelated sepsis -critical level of quantitative tip cultures. Arch. Intern. Med., $\quad$ 147: 873. http://dx.doi.org/10.1001/archinte.198 7.00370050069012

CDC/NHSN. Central Line-Associated Bloodstream Infection (CLABSI). http://www.cdc.gov/nhsn/pdfs/pscman ual/4psc_clabscurrent.pdf (Accessed 31 May 2013)

Clinical Laboratory Standard Institute (CLSI). 2008. Performance standards for antimicrobial susceptibility testing. $18^{\text {th }}$ International Supplement. CLSI document M 100- 18. Wayne, Pennsylvania.

CLSI. 2003. Method for Antifungal Disk Diffusion Susceptibility Testing of Yeasts; Proposed Guideline. CLSI document M44-P [ISBN 1-56238-4880]. CLSI, Pennsylvania, USA.

Gil, R.T., Kruse, J.A., Thill-Baharozian, M.C., Carlosn, R.W. 1989. Triple- vs single-lumen central venous catheters. A prospective study in a critically ill population. Arch. Intern. Med., 149: 1139-1143.

Heard, S.O., Wagle, M., Vijayakumar, E., et al. 1998. Influence of triple-lumen central venous catheters coated with chlorhexidine and silver sulfadiazine on the incidnece of catheter-related bacteremia. Arch. Intern. Med., 158: 81-87.

Karpel, E., Kunsdorf-Wnuk, A., Musiol, E., Skorupa, A., Arct-Danielak, D., Jarosz, U. 2006. Catheter related blood stream infection in ICU patients with prolonged central venous catheterisation-cause and prevention. Pol Merkur Lekarski, 21(123): 211217.

Meadows, C., Creagh-Grown, B., Nia, T., Bonnici, K., Finney, S. 2009. Definition of catheter-related bloodstream infection as a quality improvement measure in intensive care. Critical Care, 13: 191. http://dx.doi.org/10.1186/ cc7355

Mermel, L.A. 2000. Prevention of intravascular catheter-related infections. Ann. Intern. Med., 132: 391-402.

http://dx.doi.org/10.7326/0003-4819132-5-200003070-00009

Moro, M.L., Vigano, E.F., Cozi Lepri, A. 1994. Risk factors for central venous catheter-related infections in surgical and intensive care units. The Central Venous Catheter Related Infections 
Study Group. Infect. Control Hosp. Epidemiol., 15: 253-264.

National Nosocomial Infections

Surveillance System (NNIS). 2004.

System report, data summary from January 1992 through June 2004, issued October 2004. Am. J. Infect. Control, 32: 470-485. http://dx.doi.org/10.1016/j.ajic.2004.1 0.001

Pawar, M., Mehta, Y., Kapoor, P., Sharma, J., Gupta, A., Trehan, N. 2004. Central venous catheter-related blood stream infections: incidence, risk factors, outcome, and associated pathogens. $J$. Cardiothorac. Vasc. Anesth., 18: 304308.
Richet, H., Hubert, B., Nitemberg, G., et al. 1990. Prospective multicenter study of vascular-catheter-related complications and risk factos for positive central-catheter cultures in intensive care unit patients. J. Clin. Microbiol., 28: 2520-2525.

Tullu, M.S., Deshmuh, C.T., Baveja, S.M. 1998. Bacterial profile and antimicrobial susceptibility pattern in catheter related nosocomial infection. J. Postgrad. Med., 44(1): 7-13.

Yilmaz, G., Koksal, I., Aydin, K., Caylan, R., Sucu, N., Aksoy, R. 2007. Risk factors of catheter-related bloodstream infections in parenteral nutrition catheterization. J. Parenter. Enteral. Nutr., 31: 284-287.

\section{How to cite this article:}

Naveen, G., G. Latha and Nagraj, C. 2016. Bacteriological Study of Central Line Associated Blood Stream Infection at a Tertiary Care Hospital. Int.J.Curr.Microbiol.App.Sci. 5(9): 645649. doi: http://dx.doi.org/10.20546/ijcmas.2016.509.074 